\title{
Recherche et engagement : de l'identité nationale au paria
}

\author{
Entretien avec Laurent Bazin \\ Par François Brun
}

Pré-publication d'un article publié dans :

Migrations société, vol. 29, n 170, 2017, pages 119-133.

\begin{abstract}
Auteurs
Laurent Bazin est anthropologue au CNRS, Centre lilois d'études et de recherches sociologiques et économiques (CLERSÉ), en accueil au Centre d'étude en sciences sociales sur les mondes africains, américains et asiatiques (CESSMA). Il a mené des recherches depuis vingt-cinq ans sur les transformations du travail dans le cadre de la globalisation, respectivement en Côte d'Ivoire, France, Ouzbékistan et Algérie. Il fut président de l'Association française des anthropologues (AFA) de 2006 à 2011.

François Brun est membre du Conseil scientifique de Migrations Société, ancien chercheur au sein de l'équipe "Genre, travail, mobilités » du Centre de recherches sociologiques et politiques de Paris (CRESPPA), Université Paris VIII Vincennes-Saint-Denis et Université Paris X Nanterre.
\end{abstract}




\section{Recherche et engagement : de l'identité nationale au paria}

Nous allons nous intéresser ici aux relations entre science et engagement. Entre les positions extrêmes qui, d'un côté, au nom de l'objectivité à laquelle le chercheur est astreint, les voient comme quasiment incompatibles et celles qui, à l'inverse ne décèlent pas de réelle contradiction entre l'approche militante et le respect de méthodes scientifiques, on remarque que recherche et engagement militant sont assez communément perçus comme, pour le moins, en tension. Avant d'examiner en détail les points qui génèrent cette tension, j'ai envie de te demander comment, le plus spontanément, possible, tu aborderais cette question et où tu te situerais.

Ce n'est pas parce que, dans une démarche scientifique, l'on vise à l'objectivité, que l'on échappe aux implications politiques des connaissances que l'on produit et que l'on publie. Il n'existe pas de chercheur qui ne soit pas « engagé » dans le sens où il ne peut jamais se « désengager » du sens politique de ce qu'il produit.

Prenons un exemple classique : lorsque Pierre Bourdieu entreprend ses tout premiers travaux en Algérie, il a déjà une conception très objectiviste de la sociologie, qu'il a développée dans son œuvre ultérieure. Dans Travail et travailleurs en Algérie, il commence par réfuter un article de Michel Leiris qui affirmait qu'un ethnologue ne peut être objectif dans le contexte colonial et qu'il se doit d'être éthique. Pierre Bourdieu lui répond que l'impératif n'est pas éthique mais scientifique, et que, pour être objectif, l'ethnologue se doit d'analyser la situation existentielle du colonisé... qui est d'être colonisé !

Sa position est donc objectiviste, mais elle n'est pas dégagée des implications politiques de la situation (la guerre d'indépendance). Il s'oppose à l'attitude classique de l'ethnologie coloniale : il était très courant que les ethnologues prennent fait et cause pour les sociétés « indigènes" qu'ils étudiaient. Pourtant, dans leurs travaux, ces mêmes ethnologues « engagés », qui dénonçaient les abus du colonialisme, s'efforçaient de ne pas en voir les effets pour décrire ces sociétés dans une sorte de présent éternel. Ils contribuaient finalement à reproduire le socle idéologique du colonialisme.

La question de l'objectivité ne tient pas au fait de se vouloir engagé ou détaché de la politique : elle est plutôt dans la capacité à construire un objet intellectuel en menant une réflexion épistémologique sur les implications politiques de sa pratique scientifique.

En ce qui me concerne, je n'ai jamais été militant, sauf de manière très éphémère. En 1996, j'étais auprès des sans-papiers à l'église Saint-Bernard de la Chapelle, où j'ai campé la nuit durant une semaine ${ }^{1}$. J'ai également participé au Réseau éducation sans frontières quand mon fils était au collège. Enfin, j'ai essayé, sans grand succès, de mobiliser la communauté scientifique contre la création du ministère de l'Immigration, de l'Intégration, de l'Identité nationale et du Développement solidaire en 2007.

Je participe à l'animation de l'Association française des anthropologues (AFA) et de sa revue, le Journal des anthropologues, depuis vingt ans; j'ai été président de l'association pendant cinq ans. J'ai toujours défendu l'idée de prises de position politiques des anthropologues, notamment sur les processus d'altérisation, ou pour contribuer plus généralement à l'intelligibilité des configurations politiques, sociales, économiques, idéologiques, en France aussi bien que dans les pays qu'ils étudient, a fortiori lorsque surviennent des crises politiques et sociales. Ma perspective a toujours été celle d'une

\footnotetext{
${ }^{1}$ Le 28 juin 1996, plus de trois cents étrangers, dont une majorité en situation irrégulière, ont décidé d'occuper l'église de Saint-Bernard de la Chapelle, située à Paris, afin de revendiquer leur régularisation.
} 
anthropologie politique, dans les deux sens que cela implique: l'analyse politique des situations que j'étudie, et la restitution de cette analyse politique qui est en soi une prise de position.

Ce principe est défendu collectivement à l'AFA, mais il a fallu se battre contre un académisme disciplinaire dominant, qui a continué à reproduire, jusqu'à la fin du $\mathrm{XX}^{\mathrm{e}}$ siècle, l'aveuglement au politique de l'ethnographie coloniale. Nous avons contribué de manière décisive à faire bouger les lignes dans la discipline et à faire reconnaître la légitimité de l'anthropologie à s'intéresser aux enjeux sociaux contemporains. D'une certaine façon, pour un chercheur, la lutte et l'engagement politiques commencent dans le champ scientifique luimême.

Quant à parvenir à faire entendre sa voix au-delà du cercle restreint des sérails académiques... c'est bien plus compliqué. Je m'y suis efforcé, sans grand succès, en multipliant des initiatives, comme l'organisation de conférences ou de présentations publiques de livres. Depuis un an, je me suis lancé dans une expérience toute nouvelle pour moi, résolument militante, en soutenant le combat d'un ami spolié de son droit de séjour en France. Nous avons engagé ensemble l'écriture de son récit autobiographique, et créé un site internet pour exposer ce travail d'écriture en cours, de sorte que les mots soient son arme de combat et le porte-drapeau de nombreux autres combats ${ }^{2}$. C'est devenu une aventure collective, car nous venons de créer une association.

Me situant moi-même ouvertement du côté de ceux qui doutent de la possibilité de toujours conserver vis-à-vis de son objet la distance que "l'objectivité » est généralement supposée requérir, j'ai envie de te reposer la question en termes délibérément provocateurs : la "neutralité axiologique » n'est-elle pas, au fond, une forme de schizophrénie?

Une partie de la réponse est dans ce que je viens de dire : il n'y a pas de neutralité possible dans le champ des sciences sociales, mais ce n'est pas le principe de neutralité axiologique qui est en cause. Ce qui relèverait de la schizophrénie, ce serait plutôt la démarche ethnographique que je viens d'évoquer, et qui consiste à construire un objet scientifique en l'amputant de sa dimension politique, tout en dénonçant par ailleurs un système politique d'oppression.

La neutralité axiologique n'est pas l'absence de position politique du chercheur, mais le fait de s'abstenir de porter des jugements de valeur sur les phénomènes qu'il étudie. L'idée de Max Weber peut se résumer ainsi : si je veux comprendre un univers social particulier, ce qui inclut de comprendre son système de valeurs, je dois éviter de le regarder avec mon propre système de valeurs. Cela étant, ce n'est pas parce que j'essaye de saisir la manière d'agir et de raisonner de « l'autre » que je renonce à mes propres convictions. C'est une posture préalable à l'engagement de toute entreprise de connaissance.

La vraie question est d'ordre épistémologique. Un autre exemple : ce 23 octobre 2017, Françoise Héritier raconte, sur France inter, que dans les années 1970, les régions sahéliennes étaient touchées par la famine. Au sein du Laboratoire d'anthropologie sociale (LAS), ceux qui travaillaient sur ces zones collectaient de l'argent pour l'envoyer dans les villages qu'ils connaissaient, afin que les gens ne meurent pas de faim. Michel Izard est allé demander à Claude Lévi-Strauss s'il voulait cotiser. Ce dernier lui aurait répondu que l'important n'était pas de sauver des personnes, mais de décrire leur culture. Il s'agit là d'une parfaite illustration du recouvrement de ses orientations épistémologique et politique. L'anthropologie structurale de Claude Lévi-Strauss fait disparaître les acteurs, les sujets et les rapports sociaux derrière les structures symboliques. Il était engagé, notamment aux côtés de l'Unesco depuis l'écriture

\footnotetext{
${ }^{2}$ Voir plus loin.
} 
de son ouvrage Race et histoire en 1952 ; il a contribué à instituer les notions de patrimoine culturel et immatériel, à célébrer la diversité culturelle, et à diffuser le mot d'ordre d'ethnographie d'urgence : il fallait se dépêcher de mettre en musée les cultures et les langues menacées de disparition. Le Musée Jacques Chirac/Quai Branly, à Paris, est une réalisation actualisée de cette vision: est-ce que les «cultures»y «dialoguent», comme le dit son slogan publicitaire? Le choix a été fait d'effacer tout trace des sociétés derrière les objets présentés, et bien sûr de faire disparaître toute réflexion sur les rapports de domination dans le cadre desquels ces objets ont été collectés, et sur leur actualisation. Les « cultures » dont on vante la diversité sont en fait réduites au silence ${ }^{3}$. C'est la fonction idéologique de la diversité que d'occulter les rapports sociopolitiques.

À l'époque à laquelle se déroule l'anecdote rapportée par Françoise Héritier, le séminaire de Claude Meillassoux a abouti à une publication collective : Qui se nourrit de la famine en Afrique? Face à une situation dramatique, il y a ici trois sortes d'attitudes « engagées » : celui qui détourne la tête parce qu'il poursuit des objectifs plus «nobles»; ceux qui réagissent par une action immédiate sans remettre en cause leur objet scientifique (on peut continuer à spéculer sur l'alliance et la filiation comme si de rien n'était); ceux qui investissent leurs connaissances dans l'analyse des causes de la situation pour les dénoncer. Personnellement, c'est cette dernière démarche, la plus délibérément politique, qui me semble aussi la plus intéressante sur le plan scientifique. C'est donc au niveau épistémologique qu'il faut réfléchir: de quelle manière la position scientifique d'un chercheur s'inscrit dans le contexte politique global de son époque?

Enfin, l'observation ne peut jamais être neutre du point de vue de ceux qui en sont «l'objet». Lorsqu'un chercheur fait irruption dans un champ social pour le connaître, sa démarche devient un enjeu de ce champ social. Cet enjeu a forcément une dimension politique. Pour analyser ce qu'il observe, il doit comprendre cet enjeu, et donc analyser la position qu'il occupe dans ce champ social. Il lui faut alors analyser la nature de la relation qui s'instaure entre lui-même et les sujets qu'il rencontre sur son terrain. Or, cette relation est complexe : dans le cas de l'enquête ethnologique, elle est une interaction directe avec les sujets, mais elle s'inscrit dans les rapports globaux de domination.

Ce qui importe pour un chercheur, c'est de comprendre le monde du point de vue de ceux qu'il étudie, et non de son propre point de vue. En ce sens, le principal piège de l'engagement c'est peut-être lorsque le chercheur masque le point de vue que les « dominés » ont sur les rapports de domination en les forçant à entrer dans un cadre d'analyse théorique et politique préétabli. C'est ce qu'on peut appeler le dogmatisme théorique: l'inverse de l'empirisme ethnographique. C'était par exemple typiquement la posture des intellectuels révolutionnaires de différentes obédiences dans les années 1970, qui projetaient sur les prolétariats ou les paysanneries du tiers-monde leurs propres désirs de révolution. C'est le cas aujourd'hui sur des sujets polémiques comme la prostitution ou le voile islamique : on invalide le point de vue de celles qui sont concernées au motif qu'elles seraient aliénées. Ce faisant, on leur nie la faculté d'être des sujets politiques, ce qui revient à replier sur elles le caractère universel de la domination masculine.

Si l'on est prêt à admettre qu'engagement et recherche risquent nécessairement de se trouver à certains moments au moins en tension, notamment dans le domaine des migrations, ne doiton pas se poser la question de la subsidiarité? Le chercheur peut-il, au nom d'une sorte d'« impératif catégorique», non pas bien sûr "truquer» ses résultats, mais suspendre éventuellement des travaux dont il a lieu de craindre que les résultats ne le mettent en porteà-faux à l'égard de ses valeurs fondamentales (ce serait là, d'une certaine manière, poser le

${ }^{3}$ BAZIN Laurent, «Anthropologie, patrimoine industriel et mémoire ouvrière. Vers une recontextualisation critique », L'Homme et la société, n 192, 2014, pp. 143-166. 
principe de la subsidiarité du champ scientifique) ? Ou doit-il, au contraire, admettre a priori que les résultats de sa recherche peuvent le conduire à remettre en cause des valeurs auxquelles il était profondément attaché et qui étaient au fondement de son engagement?

Je rejoindrais tout à fait Pierre Bourdieu que je citais plus haut : l'impératif catégorique, pour un chercheur, est la rigueur de son travail scientifique et la qualité de ses travaux. Un scientifique a un "devoir de vérité ». Au sens philosophique et déontologique du terme, évidemment, et non pas au sens où il serait détenteur de "la vérité ». Pour le reste, c'est à chacun de s'arranger avec ses convictions et ses valeurs, selon ce que lui dicte sa conscience.

$\mathrm{Au}$ terme d'un travail de recherche, un chercheur aura forcément changé le regard qu'il porte sur le phénomène qu'il étudie. Dans le cas contraire, c'est que quelque chose ne va pas : soit il connaissait à l'avance les résultats et il était inutile de mener une recherche, soit il n'a rien vu et n'a rien compris à ce qu'il étudiait. Toute construction d'un objet intellectuel est simultanément un travail sur soi-même, de manière à se détacher du rapport que l'on a $a$ priori avec cet objet.

Par ailleurs, mener une recherche sur un groupe social quelconque aboutit forcément à en analyser les contradictions, les conflits, les failles. Si l'on éprouve de la sympathie pour un mouvement social, ça ne me semble pas très avisé de le prendre pour objet de recherche, a fortiori quand on y milite, à moins que l'objectif soit précisément d'en éclairer les contradictions pour contribuer à leur résolution. C'est la démarche de l'analyse institutionnelle, par exemple.

En revanche, si ce qu'on découvre dans un travail de recherche est déplaisant, je crois qu'il faut malgré tout aller jusqu'au bout. Il n'y a rien de pire pour un chercheur que d'occulter une partie de la réalité, de la tronquer, c'est-à-dire de s'autocensurer ou de faire preuve de mauvaise foi dans l'argumentation scientifique, consciemment ou inconsciemment, que ce soit pour sauvegarder ses propres convictions politiques et/ou idéologiques, ou bien pour tenter de préserver l'image ou les intérêts de tel ou tel groupe social qu'il étudie. Si son argumentation n'est pas solide et suffisamment étayée, elle sera facilement attaquée par ses adversaires politiques et/ou scientifiques. Au final, il ne rendra pas service à la cause qu'il défend.

Les migrations m'ont toujours semblé un sujet difficile, parce qu'entièrement surdéterminé par le politique. Il faut rappeler un vieux débat qui agitait l'ethnologie urbaine naissante dans les années 1970. Gérard Althabe et Monique Selim s'opposaient à certains ethnologues qui, considérant que leur discipline avait pour vocation à étudier " l'autre ", choisissaient pour objet l'une des figures de «l'autre» au sein de la société française ; en faisant cela, ils contribuaient en quelque sorte à réifier cette altérité. Gérard Althabe et Monique Selim ont été, au contraire, des précurseurs dans l'analyse de ce qu'ils appelaient « la production de l'étranger» : à partir d'enquêtes dans des quartier urbains, ils montraient que la figure de l'étranger était une production imaginaire centrale dans les configurations locales des rapports sociaux.

Le problème posé est en fait inhérent à toute forme de délimitation d'un terrain d'étude et d'un objet : il y a toujours un risque de surdétermination. On s'extasie devant une belle œuvre ethnographique sur la banlieue ou la prison, par exemple, où l'auteur fournit une description très fine des codes langagiers, gestuels, vestimentaires, etc. Le terrain est décrit comme une sous-culture, un univers doté de ses propres valeurs et de ses propres normes, clos sur lui-même. L'effet peut être encore renforcé si l'observateur est lui-même partie prenante de ce monde, s'il en est issu ou occupe une fonction quelconque comme enseignant, éducateur, etc. L'ethnographe peut être engagé auprès des acteurs qu'il étudie - s'insurger de la stigmatisation dont les banlieues sont l'objet par exemple - et participer au renforcement des processus globaux d'exclusion. Dans ce cas, oui, il y a tension entre recherche et 
engagement, mais l'auteur ne la perçoit pas forcément sous cet angle, car il ne voit pas qu'il est en train de participer à l'altérisation de son objet, à son exotisation.

Or, cette altérisation est aujourd'hui au cœur des enjeux politiques de la société française, comme partout ailleurs. Un autre objet piégé par excellence est bien sûr l'islam, aujourd'hui placé au centre de multiples conflits, à la fois en France et au niveau global.

La question peut être posée en termes de temporalité. L'idée serait qu'on peut être chercheur et militant, mais pas au même moment. On aurait en quelque sorte plusieurs casquettes: tantôt, on porterait celle du chercheur, tantôt celle du militant? En ce qui concerne les politiques migratoires actuelles et leur mise ouvre au quotidien, les occasions ne manquent pas de prendre position, mais dans quelle mesure est-on fondé à le faire en tant que chercheur?

Il y a de multiples façons d'être militant et de multiples façons de faire de la recherche : je ne crois pas qu'il $\mathrm{y}$ ait de règle générale. Je ne crois pas non plus que les chercheurs fonctionnent tous selon les mêmes rythmes.

Sur un certain nombre de questions politiques, au sens où elles concernent la société tout entière et son mode de gouvernement, les chercheurs ont une responsabilité : celle de connaître et de faire connaître, de produire un éclairage sur la complexité du monde. Les universitaires et les chercheurs sont employés par l'État, mais ils ne sont pas au service de l'État : ils sont au service du public. Une loi récente fait obligation aux chercheurs de mettre leurs publications en accès libre sur internet un an après leur parution. De tous les secteurs de la fonction publique, les universitaires et les chercheurs sont les seuls qui ne sont pas astreints au devoir de réserve : ils ont un devoir de vérité, et ce devoir de vérité s'exerce aussi à l'encontre des politiques étatiques et des dispositifs du pouvoir et de l'autorité.

Il me semble fondamental aujourd'hui de s'exprimer, d'«ouvrir sa gueule». Et notamment sur les questions que l'on appelle «migratoires ». Il s'agit d'un euphémisme, car le cœur du problème ce ne sont nullement les migrations, mais bien les politiques antimigratoires, menées tant au niveau national qu'européen. Ces politiques sont criminelles, elles tuent des milliers de personnes, condamnent des centaines de milliers d'autres à une existence de parias, et disloquent les sociétés, en Europe comme dans son " entourage », car elles participent évidemment de la déstabilisation de l'ensemble de l'Afrique et du MoyenOrient.

Est-ce que les chercheurs sont fondés à prendre position ? C'est leur rôle ! Néanmoins, d'une part, il ne faut pas se faire trop d'illusion sur leur capacité à peser dans le débat public. La marginalisation de la science dans l'économie politique de la communication est un objet de réflexion fondamental. D'autre part, un chercheur ne peut se contenter de reprendre les termes des débats politiques. Son rôle est de les inclure dans sa réflexion, d'en éclairer les significations, de déconstruire les évidences et de restituer la complexité du réel, notamment en décryptant les liens entre des phénomènes que le débat public considère séparément.

Il y a, dans les postures que les chercheurs peuvent être conduits à adopter, un aspect qui est certainement de moins en moins négligeable: c'est tout bêtement celui de la carrière et, même plus largement de l'emploi et de la publication: un chercheur qui affiche ses engagements ne s'expose-t-il pas à une perte de crédibilité et, partant, à avoir plus de mal à être embauché, à publier (et à choisir ses supports de publication), à progresser en répondant aux critères dits "d'excellence " qui sont de plus en plus de mise aujourd'hui ? Et, à l'inverse, ne risque-t-il pas d'être tenté par une carrière médiatique pour laquelle il lui serait profitable de faire état de ses engagements, quitte à les instrumentaliser? 
Cela dépend des types d'engagement, de la hiérarchie de prestige des objets, des disciplines, etc. Il y a des sujets et des engagements "maudits» et d'autres qui sont au contraire très valorisés. Une partie de «l'élite » de l'EHESS collabore avec des think tanks où s'élabore le « réformisme de gauche ». Leur collusion avec des acteurs politiques et syndicaux en position de décideurs ne dévalorise en rien ces chercheurs, bien au contraire : ils gagnent honneurs, distinctions, promotions, publicisation de leurs travaux, attention des médias, rétributions matérielles, reconnaissance institutionnelle et... « scientifique ».

À l'évidence, d'autres se positionnent et se valorisent sur le marché de la contestation et en deviennent des vedettes, avec ce que cela suppose également comme rétributions matérielles et symboliques. Ceux qui sont probablement assurés d'une plus grande notoriété sont ceux qui se spécialisent dans l'importation des courants critiques nord-américains.

Un chercheur militant qui investit ses recherches et ses réflexions sur des causes plus marginales risque beaucoup plus d'être lui-même marginalisé. Les auteures féministes par exemple, dans les années 1970-80, ont eu beaucoup de mal à obtenir une reconnaissance, et c'est probablement encore le cas aujourd'hui, malgré l'institutionnalisation dont bénéficie le "genre» et l'affichage de l'égalité hommes-femmes comme «valeur républicaine ». Pourtant, la critique féministe, peut-être surtout la plus radicale, a apporté une contribution majeure à la connaissance, en termes à la fois empiriques, théoriques et épistémologiques.

Aujourd'hui, la compétition pour l'accès aux métiers de la recherche et de l'enseignement supérieur a, en toute hypothèse, un effet de neutralisation des engagements. De plus, il y a une véritable normalisation des institutions et des parcours, ce qui rend sans doute les profils de recrutement plus homogènes.

Dans les années 1960-1970, les universités se sont scindées en suivant souvent des lignes de clivage politiques; aujourd'hui elles fusionnent pour se positionner sur le marché global : il faut peser dans le classement de Shanghai, même si tout le monde sait qu'il est «bidon ». Nos institutions sont devenues le siège d'une imitation parodique du monde du capitalisme globalisé, mais il s'agit d'un marché de dupes, ce que nous avions appelé avec Monique Selim en 2001, un «mime du marché » ${ }^{4}$. En revanche, les effets de normalisation en sont tout à fait tangibles : j'ai publié précisément un article sur la soumission du travail scientifique aux normes du marché ${ }^{5}$, vers lequel je me permets de renvoyer.

Toutefois, cela est très variable d'une discipline à l'autre. C'est en économie que les effets de normalisation sont les plus drastiques : les " hétérodoxes » se plaignent de ne plus pouvoir accéder à une reconnaissance institutionnelle et, pour les jeunes docteurs, de ne plus pouvoir accéder aux postes. Ils ont échoué à créer une section autonome au sein du Comité national du Centre national de la recherche scientifique (CNRS). L'existence de l'école de la régulation, un courant de pensée dynamique en France, est à terme menacée. Les " économistes attérés » ont une raison supplémentaire de l'être : ils sont progressivement évacués du champ de bataille que constitue leur discipline.

Tu as notamment eu constamment à t'interroger sur la notion d' "identité »? Or, celle-ci, par l'usage qui en est fait, particulièrement ces dernières années dans le discours politique est particulièrement chargée? Comment considères-tu que le chercheur doit l'aborder? En 2007, a été créé un "Ministère de l'Immigration, de l'Intégration, de l'Identité nationale et du Codéveloppement». Cet intitulé a choqué, bien au-delà des associations qui défendent les droits des migrants, et a notamment suscité une mobilisation ès qualités de réseaux scientifiques Peux-tu revenir sur cet épisode?

${ }^{4}$ BAZIN, Laurent; SELIM, Monique, «Présentation. Mimes et pantomimes du marché », Histoire \& Anthropologie, $\mathrm{n}^{\circ} 22,1^{\mathrm{er}}$ semestre 2001, pp. 11-21.

${ }^{5}$ BAZIN, Laurent, « Normes du travail scientifique et normes du marché », Journal des anthropologues, $\mathrm{n}^{\circ} 136$ 137, 2014, pp. 7-18. 
L'identité m'a toujours semblé une très mauvaise notion : elle est omniprésente dans les travaux scientifiques contemporains, mais personne n'est capable d'en donner une définition satisfaisante. C'est ce que démontrent très clairement Frederick Cooper et Rogers Brubaker dans un article critique ${ }^{6}$. C'est malgré tout une notion qui a envahi la politique et les rapports sociaux, partout, et c'est donc sur cela qu'il faut s'interroger. Je m'y suis intéressé à plusieurs reprises en analysant l'identité comme une production idéologique du marché et de la consommation.

Ce sont ensuite les idéologies de l'identité nationale que j'ai essayé d'analyser. J'étais en Côte d'Ivoire en décembre 1994 quand le gouvernement a promulgué un nouveau code électoral qui a lancé les polémiques sur «l'ivoirité ». Dès son introduction, cette notion a envahi le débat politique, et a agi comme un catalyseur des conflits. Elle est demeurée centrale durant toute la période de guerre entre 2002 et 2011. Les tensions qu'elle a créées ne sont pas réglées aujourd'hui.

J'ai travaillé sur l'Ouzbékistan de 2003 à 2006. Le régime est une dictature particulièrement oppressive, qui terrorise la population. À l'indépendance, en 1991, il est passé du communisme à une nouvelle idéologie d'État: l'identité nationale. La notion d'idéologie d'État est héritée de l'Union des républiques socialistes soviétiques (URSS) : elle est conçue comme un produit scientifique élaboré sous l'égide de la philosophie. L'État mobilise donc les chercheurs de toutes les disciplines pour produire cette idéologie de l'identité nationale. Parallèlement, l'ouzbékisation de l'État a entraîné une retraditionnalisation de la société et une obsession pour ce que l'on pourrait désigner les «normes de l'identité nationale » (o'zbekchilik) qui exercent une véritable tyrannie : c'est ce que j'observais sur mon terrain.

J'étais à peine rentré en France que Nicolas Sarkozy a lancé sa campagne électorale pour les élections présidentielles de 2007 sur le thème de l'identité nationale. Cela m'a fait un choc terrible. Élu, il a instauré un ministère « de l'Identité nationale ».

J'étais président de l'Association française des anthropologues à ce moment. J'ai alors proposé au bureau de l'AFA de publier un communiqué sur le sujet, puis d'organiser une assemblée pour débattre de la situation, en invitant les collectifs d'universitaires qui avaient exprimé leur opposition à ce ministère: notamment le réseau Terra et les historiens démissionnaires du conseil scientifique du Musée national de l'histoire de l'immigration. L'assemblée s'est tenue dans le grand amphithéâtre de l'EHESS, qui était plein. Il a alors été décidé de créer un mouvement de chercheurs, que nous devions coordonner avec Jérôme Valluy (Terra), mais tout cela s'est disloqué très vite. J'étais naïf et $\mathrm{j}$ 'ai appris à mes dépens à quel point il était difficile de mobiliser le milieu des chercheurs et des universitaires.

Avec l'AFA, nous avons organisé un colloque «identités nationales d'État » et publié un numéro hors-série du Journal des anthropologues. Le point de vue anthropologique que nous avons essayé de faire valoir soulignait la dimension mondiale du phénomène d'identité nationale, et son caractère multisectoriel, ce qui permettait de porter un autre regard sur la situation française ${ }^{7}$.

Des journalistes sont venus à l'assemblée que j'avais organisée et ont fait paraître plusieurs interviews de sociologues ou d'historiens de l'immigration. Une journaliste de Libération a fini par m'interviewer également par téléphone, ce qui a donné lieu à... cinq lignes dans le journal. Je ne conteste évidemment ni la pertinence ni la justesse de la contribution des sociologues et historiens de l'immigration sur la question, mais c'est un

\footnotetext{
${ }^{6}$ BRUBAKER, Rogers ; COOPER, Frederik, « Identité », in : COOPER, Frederik, Le colonialisme en question. Théorie, connaissance, histoire, Paris :Éd. Payot, 2010, pp. 81-123.

${ }^{7}$ BAZIN, Laurent ; GIBB, Robert ; SELIM Monique, « Nationalisation et étatisation des identités dans le monde contemporain », Journal des anthropologues, $\mathrm{n}^{\circ}$ hors-série “Identités nationales d'État", 2007, pp. 7-35.
} 
piège médiatique redoutable, car le débat restait enfermé sur la question des «immigrés », que pointaient d'ailleurs l'intitulé du ministère «Immigration, Intégration, Identité nationale » et son organisation (l'administration des étrangers). Or, « étrangers » et « immigrés » sont des tiers dans cette production idéologique de l'identité nationale. Précisément des tiers exclus ! Et ce ne sont pas n'importe quels « étrangers » d'ailleurs. Le "grand débat » sur l'identité nationale, ordonné en 2010 par le ministre de l'époque, s'est focalisé exclusivement sur l'exclusion de l'islam de l'identité nationale. Cela étant, ce résultat n'était en définitive que la réplique de ce que produisaient quotidiennement les plateaux de télévision depuis trois décennies. C'est sans doute pourquoi le thème de l'identité nationale a fini par être délaissé au profit du seul culte de la République.

Mon propos était trop en décalage pour être entendu. Je suis parvenu à publier en 2010 un article dans le Monde diplomatique où je compare la Côte d'Ivoire et l'Ouzbékistan ${ }^{8}$, en contrepoint du «grand débat». Dans un article plus approfondi je compare les trois pays : France, Ouzbékistan et Côte d'Ivoire ${ }^{9}$. Une telle comparaison a une dimension subversive, car elle dissout les raisonnements dichotomiques entre "nous» et "eux » qui continuent à structurer très fortement la pensée scientifique autant que les perceptions ordinaires : personne n'a envie de voir des processus similaires derrière la dictature ubuesque de l'Ouzbékistan et la démocratie du pays des droits de l'homme. Elle est subversive aussi car elle permet de recentrer justement l'attention, non sur les "étrangers » et leur « intégration », mais sur la manière dont l'État se redéfinit dans la conjoncture de globalisation néolibérale, en modifiant les conceptions de la citoyenneté. Celle-ci n'est plus définie autour d'un projet politique de modernisation mais sur l'idée d'une population autochtone à défendre contre des menaces extérieures. En France, c'est l'identification aux «valeurs de la République » qui devient une ligne de séparation imaginaire entre un «nous» autochtone et des " autres » menaçants, venus d'ailleurs. Cela étant, en France, comme en Ouzbékistan et en Côte d'Ivoire, une telle délimitation ne déstabilise pas seulement les fractions sociales qu'elle désigne comme des étrangers intérieurs : elle dés-intègre toute la société dans sa globalité, parce que, pour le dire de manière imagée, « nous sommes tous des enfants d'immigrés ".

En 2008, j'ai été auditionné par la Commission sur la diversité, dont la responsabilité avait été confiée par les pouvoirs publics à Michel Wieviorka. Outre le fait d'avoir attaqué la notion de diversité, j'ai essayé de faire passer un seul message, qui me semblait s'imposer dans le climat politique créé par l'élection de Nicolas Sarkozy: pour lutter contre les discriminations qui frappent les catégories sociales d'origine étrangère (puisque ce sont d'elles dont il s'agit), il faut commencer par réduire la pression qui s'exerce à l'encontre de l'immigration, et donc s'attaquer aux discours politiques de stigmatisation des étrangers. Le membre de la commission qui s'est opposé le plus fermement à cet argument était... président d'un collectif de sans-papiers ! Il tenait au contraire à maintenir une séparation étanche entre les deux questions, c'est-à-dire entre les deux catégories de population définies juridiquement : étrangers et nationaux. La diversité ne concernait que les nationaux. Peut-être sa vision était-elle plus juridique que politique ? En cela elle se conformait d'ailleurs à la commande politique $^{10}$. Mais si la désignation d'étranger s'arrêtait avec l'acquisition de la citoyenneté française, il n’y aurait pas besoin de s'inquiéter de diversité ; on ne pourrait pas non plus exclure les étrangers des questions de la lutte contre les discriminations. Mon

\footnotetext{
${ }^{8}$ BAZIN, Laurent, «La pandémie de l'identité nationale. Aucun vaccin disponible à ce jour », Le Monde Diplomatique, $\mathrm{n}^{\circ}$ 671, février 2010, p. 3.

9 BAZIN, Laurent, «Idéologies de l'identité nationale et formes de citoyenneté. Une réflexion comparative (Côte d'Ivoire, France, Ouzbékistan) », in : TOLAN, John ; EL ANNABI, Hassen ; LEBDAI, Benaouda ; LAURENT, Franck ; KRAUSE, Günter (sous la directionde), Enjeux identitaires en mutations. Europe et bassin méditerranéen, Berne : Peter Lang, pp. 35-60.

10 Voir la lettre de mission publiée dans: VIEWIORKA, Michel, La diversité 'rapport à la Ministre et l'enseignement Supérieur et de la Recherche sur), Paris : Éditions Robert Laffont, 2008, 228 p.
} 
argument a évidemment été évacué : le rapport rendu public se garde bien de toute allusion à la politique de restriction des migrations, aux discours stigmatisants qui l'accompagnent, à la politique de l'identité nationale. Un accommodement raisonnable, en somme.

$T u$ as récemment créé une association dont l'un objets est de multiplier les passerelles entre la "communauté » scientifique et l'univers militant, et également les formes d'expression artistique. Peux-tu nous en parler plus précisément?

Nous venons de créer une association: Le paria. C'est une association qui se donne pour but d'articuler l'action politique et militante, le registre scientifique et la recherche de modes d'expression artistique.

À l'origine, se trouve un projet d'ouvrage que j'ai entrepris d'écrire avec Mohamed Bridji, que j'ai rencontré à Oran : il avait perdu son titre de séjour en France à la suite d'une incarcération et il avait été expulsé en Algérie. Il avait quitté ce pays en 1990, à l'âge de sept ans, et n'y était revenu qu'une seule fois en vacances. Je lui ai apporté mon soutien, et je me suis efforcé de l'aider à sortir d'une situation d'exclusion radicale. Je me suis investi avec lui dans un combat pour qu'il soit rétabli dans ses droits en France.

Nous avons engagé l'écriture d'un livre, Le retour du refoulé. C'est le récit de sa vie, mais nous ne l'avons pas conçu comme un simple recueil d'une autobiographie par un chercheur. Nous voulions en faire un livre qui permette à Mohamed de faire entendre sa voix propre, par mon intermédiaire, puisque c'est moi qui tiens la plume. Il est mon co-auteur, et non mon «objet d'étude». L'objectif était de mettre son histoire sur la place publique, et d'emprunter une forme d'écriture littéraire pour toucher un public le plus vaste possible.

En mai 2016, Moha - c'est le nom de son personnage dans son récit, clin d'œil à Moha le fou, Moha le sage 11 - a déposé une demande de titre de séjour en France ; en juin, nous avons entamé la rédaction du livre. En octobre, il a reçu un refus de titre de séjour, accompagné d'une obligation de quitter le territoire. Nous avons engagé un recours devant le tribunal administratif et, dans le même temps, nous avons bâti un site internet, Leparia.fr, pour faire connaître le travail d'écriture en cours. L'objectif premier était de publiciser l'histoire de Moha sans attendre la publication du livre, et de bâtir un réseau de soutien pour l'appuyer dans ses démarches juridiques et prendre sa défense s'il devait à nouveau être menacé d'expulsion.

La création du site internet a conféré à notre travail une autre dimension, car les technologies numériques permettent d'explorer des formes d'écriture originales, qui sont encore à inventer. Notre démarche a inspiré d'autres personnes qui sont venues s'associer à notre action, chacune à sa manière, avec ses motifs propres et ses projets : le duo initial est devenu un collectif, puis une association. Au cœur de cette démarche, il y a l'exploration de modes d'action, de connaissance et d'expression orientés vers des objectifs politiques. C'est cela que l'on essaie de poursuivre collectivement.

Quant à Moha, il a gagné son procès et vient de recevoir sa carte de séjour.

\section{Un mot de conclusion?}

J'ai essayé de dire qu'il n'y a pas de science possible sans engagement. Peut-être parce que nous vivons dans un monde où l' «État-inconscient », pour reprendre les mots de René Lourau, est profondément ancré. Quel que soit ce qu'il observe, la manière dont il l'observe, et où, un chercheur rencontrera forcément l'État. Le sien. Les autres. Le monde. S'il se veut détaché de la politique, c'est qu'il voit un monde tronqué. Cela se comprend évidemment

\footnotetext{
${ }^{11}$ BEN JELLOUN, Tahar, Moha le fou, Moha le sage, Paris : Éditions du Seuil, 1978, 185 p.
} 
dans des contextes où la critique politique est frappée d'interdit: mes collègues d'Ouzbékistan craignent pour leur vie ; ceux de Côte d'Ivoire surveillent leurs mots, ce qui n'était pas le cas dans les années 1990 ; en Algérie la critique est aujourd'hui bien plus libre, mais le régime se durcit actuellement. Toute production de connaissances qui se veut « objective » en sciences sociales contient nécessairement une analyse politique, et celle-ci ne peut se passer d'une réflexion épistémologique. Cela n'oblige personne à prendre parti, mais ça ne l'empêche pas non plus!

En ce qui me concerne, l'élection présidentielle de 2007 a été un moment de basculement, à partir duquel j'ai réellement commencé à me préoccuper de la manière de diffuser auprès du public des travaux scientifiques. Il ne s'agissait plus seulement pour moi de connaître, mais de faire connaître. J'ai essayé d'orienter l'action de l'AFA dans ce sens en tant que président, mais les résultats n'ont guère été à la hauteur de mes attentes. L'aventure engagée avec Moha par l'écriture du Retour du refoulé et du site Leparia.fr m'a permis de rebondir sur la question en m'en donnant une autre vision, parce qu'il s'agit d'articuler un propos scientifique avec une action politique concrète ; de trouver une autre manière d'écrire pour connaître et faire connaître ; de réfléchir à l'inscription des connaissances scientifiques dans une économie politique de la communication marquée par l'immédiateté, la prédominance de l'image et la profusion d'informations; et enfin de concilier différents modes de connaissance et d'expression (science, art, action politique). Cela ouvre tout un univers de possibilités à explorer. 